\title{
Large-angle magnetization dynamics investigated by vector-resolved magnetization-induced optical second-harmonic generation
}

\author{
Th. Gerrits, T. J. Silva, and J. P. Nibarger ${ }^{a)}$ \\ National Institute of Standards and Technology, Boulder, Colorado, 80305 \\ Th. Rasing \\ NSRIM Institute, Radboud University Nijmegen, 6525 ED Nijmegen, The Netherlands
}

(Received 19 July 2004; accepted 8 September 2004)

\begin{abstract}
We examine the relationship between nonlinear magnetic responses and the change in the Gilbert damping parameter $\alpha$ for patterned and unpatterned thin Permalloy films when subjected to pulsed magnetic fields. An improved magnetization-vector-resolved technique utilizing magnetization-induced optical second-harmonic generation was used to measure magnetization dynamics after pulsed-field excitation. The magnetization excitations were achieved with pulsed fields aligned parallel to the hard axis of thin permalloy $\left(\mathrm{Ni}_{80} \mathrm{Fe}_{20}\right)$ films while a dc bias field is applied along the easy axis. At low bias fields, $\alpha$ was inversely related to the bias field, but there was no significant reduction in the absolute value of the magnetization, as might be expected if there was significant spin-wave generation during the damping process. We discuss the discrepancies between data obtained by ferromagnetic resonance, whereby spin-wave generation is prevalent, and pulsed-field studies, with the conclusion that fundamental differences between the two techniques for the excitation of the ferromagnetic spin system might explain the different proclivities toward spin-wave generation manifest in these two experimental methods. (c) 2004 American Institute of Physics. [DOI: 10.1063/1.1811783]
\end{abstract}

\section{INTRODUCTION}

Since the late 1990s, the development of femtosecond laser sources and short current pulses that are much shorter than fundamental time scales, such as spin-lattice relaxation times and precessional time scales, has led to a renaissance in the study of magnetization dynamics. ${ }^{1,2}$

Generally, two approaches are used to generate the magnetic field necessary to create precessional motion.

(1) A continuous ac field drives the precessional motion near resonance, as in ferromagnetic resonance (FMR).

(2) A transient magnetic field pulse perpendicular to the magnetization (M) excites the precession.

In the stationary FMR mode, the experimental parameters are typically the direction of the applied ac microwave field, field frequency, field amplitude, and applied dc field strength, while the microwave response is the only measurable quantity. The FMR technique is susceptible to spinwave instabilities, which are manifest when the ac field amplitude exceeds the critical threshold for run-away spin wave generation. ${ }^{3}$ In contrast, the pulse technique uses the field pulse direction, the pulse shape, magnitude, duration, and the dc field strength as adjustable experimental parameters. The measurement might be as extensive as a full threedimensional description of the magnetization motion over the period of the experiment. Pure relaxation phenomena, such as free induction decay, can be directly measured by such a technique. In addition, a vector map of the dynamics can be constructed from the data. ${ }^{4-6}$

Optimal switching of magnetic elements for magnetic

${ }^{\text {a)} P r e s e n t ~ a d d r e s s: ~ S t o r a g e T e k, ~ L o u i s v i l l e, ~ C O ~} 80027$. random-access memory requires uniform excitation of the individual elements such that the entire magnetization of the element rotates coherently. Under these conditions, the magnetization dynamics may be approximated as those for a single domain ("macrospin"). In this case, pulse timing may also be used to terminate the precession. ${ }^{7}$ The motion of such a macrospin can be described by the Landau-Lifshitz-Gilbert (LLG) equation. ${ }^{8}$

$$
\frac{d \vec{M}}{d t}=-|\gamma| \mu_{0}\left(\vec{M} \times \vec{H}_{e f f}\right)+\frac{\alpha}{M_{s}}\left|\vec{M} \times \frac{d \vec{M}}{d t}\right|,
$$

where $\mathbf{H}_{\text {eff }}$ is the total effective internal field, $\alpha$ is the Gilbert damping parameter, $\gamma$ is the gyromagnetic ratio, $\mu_{0}$ is the permeability of free space, and $M_{s}$ is the saturation magnetization. The best way to achieve coherent, fast precessional motion is by applying a short-rise time, high-amplitude magnetic field pulse oriented perpendicular to the initial direction of $\mathbf{M}$. In this way, coherent excitation of the magnetization is possible, and any inhomogeneous response should be suppressed. Furthermore, the torque exerted on $\mathbf{M}$ is maximum and the reversal process occurs in the shortest possible time.

In contrast, high-power FMR experiments exhibit instabilities when the magnetization precession angle is more than a few degrees from equilibrium direction and spin-wave generation is enhanced. ${ }^{9,10}$ Patton showed ${ }^{3}$ that the smallangle magnetization motion $\left(\sim 4^{\circ}\right.$ in-plane angle) of a permalloy system, driven by high-power FMR becomes incoherent due to the occurrence of parametric excitation of $\omega / 2$ spin waves. In contrast to this result, Nibarger et al. ${ }^{11}$ have demonstrated, using a Pulsed Inductive Microwave Magnetometer (PIMM), that no change in the Gilbert damping pa- 
rameter $\alpha$ was present up to deflection angles of $40^{\circ}$ in permalloy when excited by a transient magnetic field pulse. It was also shown ${ }^{4}$ that a coherent magnetization reversal is possible at which the magnetization vector length remained unchanged. However, enhanced generation of magnons can occur for pulsed measurements if the dc bias field is applied along the hard axis, even if the bias field exceeds the anisotropy by a factor of $2 .^{5}$ This suggests that details concerning the spin-orbit coupling might have some bearing on observation of spin-wave instabilities in pulsed measurements.

Generation of spin waves leads to an increase in the measured damping parameter, as the transverse magnetization components decay faster with a rate given by the spinwave generation rate. Therefore, we expect an increase in the damping parameter correlated with an enhanced spin-wave generation. Besides an increase in the transverse relaxation rate, an increase in the magnon density also causes a reduction in the length of the magnetization vector. Vectorresolved magnetometry therefore enables one to correlate the generation of spin waves with the change in the damping parameter.

In the present paper, a correlation between the damping parameter and the time evolution of the absolute value of the magnetization $|\mathbf{M}|$ will be discussed. Time-resolved magnetization-induced optical second-Harmonic generation (MSHG) (Refs. 12 and 13), with time resolution in the subnanosecond range, is used to measure the two in-plane magnetization components in order to reconstruct $|\mathbf{M}|$ unambiguously $^{14}$ and to probe the existence of spin waves smaller than the probe spot. The dc bias field is applied along the easy axis to ensure that the magnetization is in a welldefined ground-state configuration prior to the application of the field pulse. In addition, we can directly determine $\alpha$ from the measured time trace of $\mathbf{M}$. We will show that, at low bias fields, the damping parameter $\alpha$ increases with decreasing bias field, as was already shown previously by Silva et al. ${ }^{15}$ and Klemmer et al. ${ }^{16}$ However, in the present study, no strong correlation between the increase in damping and a reduction of $|\mathbf{M}|$ could be inferred. Rotation angles up to $160^{\circ}$ were achieved, up to which point the magnetization did not change its magnitude in continuous thin films. The motion of $\mathbf{M}$ stays coherent; i.e., $|\mathbf{M}|$ does not change during rotation. This is interpreted as evidence for the absence of any significant magnon generation during large-angle, freeinduction decay of the magnetic moment.

\section{EXPERIMENTAL SETUP}

A pulse-generator excitation technique was used to study quasi-infinite magnetic thin films at very low bias fields. The magnetic fields used were steplike field pulses with maximum amplitudes of $1.23 \pm 0.02 \mathrm{kA} / \mathrm{m}(15.5 \pm 0.2 \mathrm{Oe})$, with a rise time of about $50 \mathrm{ps}$ and a duration of $10 \mathrm{~ns}$. The coupling of the magnetic field pulses to the magnetic sample was achieved by using coplanar waveguides (CPWs). The width of the center conductor of the CPW was $130 \mu \mathrm{m}$. The CPWs were matched to the pulse generator's characteristic impedance of $50 \Omega$ to minimize loss. Figure 1 shows the schematic experimental configuration. A magnetic sample is

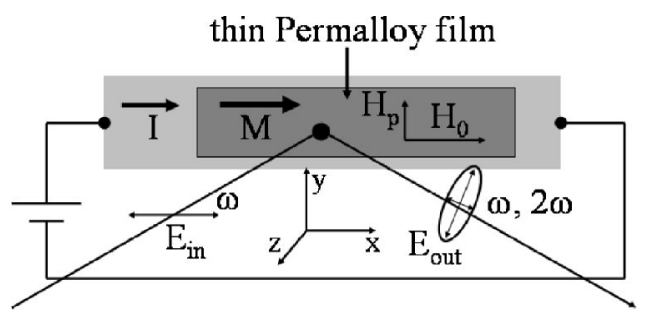

FIG. 1. Experimental scheme for magnetization precession investigations using pulsed magnetic fields generated using a commercial pulse generator. A current pulse through the center conductor of the waveguide creates a magnetic field pointing along the $y$ axis. This field excites the magnetization of a permalloy film, which initially lies along the $x$ axis. The magnetization state is probed by means of MSHG.

placed on top of a CPW structure. The magnetic field pulse is oriented along the $y$ direction such that the pulse excites the magnetization, which lies initially along the $x$ axis. The applied bias field $H_{b}$ was parallel to the anisotropy easy axis of the magnetic thin film along the $x$ axis. The laser spot on the sample was focused down to a size of $\approx 5 \mu \mathrm{m}$ in diameter. The incident polarization of the laser pulse was set parallel to the plane of incidence $p$ orientation. In the case of an isotropic interface, it can be shown ${ }^{12}$ that a rotation of the polarization of the MSHG signal can be observed due to a change in the longitudinal component of $\mathbf{M}\left(M_{x}\right)$. A change in the intensity of the MSHG signal results from a change in the transverse component of $\mathbf{M}\left(M_{y}\right)$. Therefore, both the amplitude and polarization rotation of the MSHG signal are analyzed. These two quantities have a direct and unambiguous relation to the two in-plane magnetization components, while neglecting the out-of-plane (polar) magnetization component and its contribution to the MSHG signal. ${ }^{17}$

Figure 2 shows the experimental scheme for simultaneous detection of the two in-plane components of $\mathbf{M} .{ }^{17}$ For that purpose, the signal is split into two parts with different types of signal processing. A photoelastic modulator (PEM) is used (a) to compensate for intensity changes due to polarization rotations due to MSHG and (b) to determine the amount of polarization rotation. The average photomultiplier (PMT) signal of PMT-2 is sensitive only to $M_{y}$, whereas the

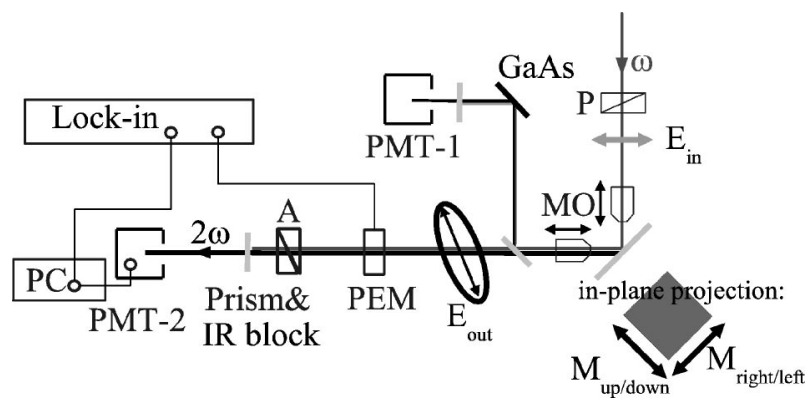

FIG. 2. Experimental configuration used to probe the two in-plane components of M. $P$, polarizer; $A$, analyzer. The average intensity is measured at photomultiplier tube 2 (PMT-2). The polarization rotation is determined by a modulation technique using a photoelastic modulator (PEM) and measuring the modulation signal by means of a lock-in amplifier. Note that the sample is rotated by $90^{\circ}$ in this figure to indicate the various magnetization directions, $M_{\text {left/right }}\left(M_{x}\right)$ and $M_{\text {up/down }}\left(M_{y}\right)$. The laser beam is focussed onto the sample by a microscope objective lens. A reference line with a GaAs sample is used to normalize the SHG signal. 

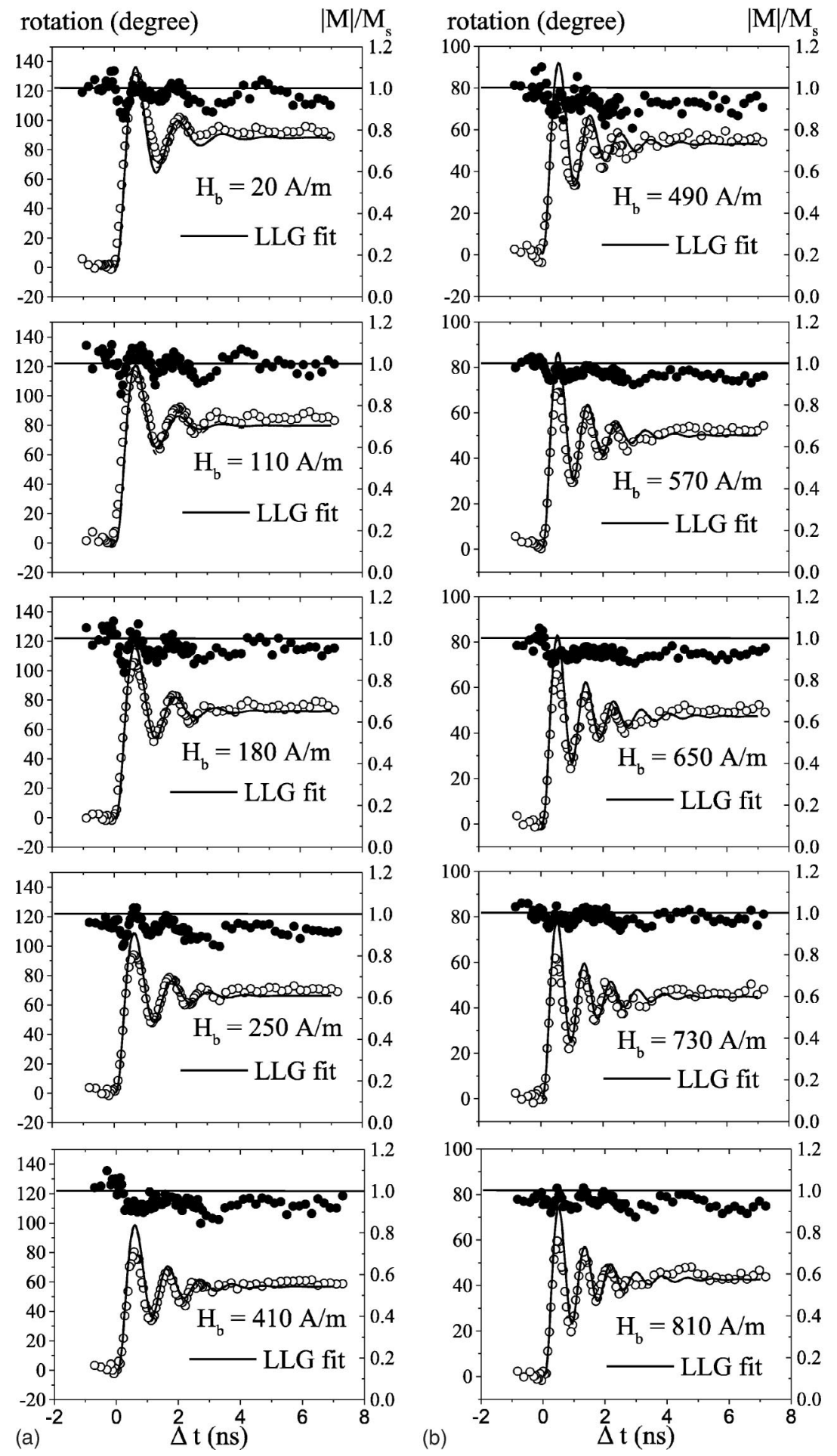

lock-in amplifier measures $M_{x}$ by detecting the amount of modulation induced by the PEM. Moreover, a normalization branch with a GaAs reference sample is introduced to compensate for both intensity fluctuation and drift in the width of the laser pulses.

\section{RESULTS AND DISCUSSION}

The data presented in Figs. 3 and 4 are obtained on a quasi-infinite $50 \mathrm{~nm}$ thin permalloy film. The boundaries of the magnetic excitation are given by the width of the center conductor of the waveguide structure, being $130 \mu \mathrm{m}$. Figure
FIG. 3. In-plane vector-resolved MSHG-measurements (open circles) with varying bias field between $H_{b}$ $=20 \pm 10 \mathrm{~A} / \mathrm{m}(0.2 \pm 0.1 \mathrm{Oe})$ and $H_{b}$ $=810 \pm 10 \mathrm{~A} / \mathrm{m}(10.2 \pm 0.1 \mathrm{Oe})$. The steplike field pulse was $H_{p}=1.23 \pm 0.02 \mathrm{kA} / \mathrm{m}(15.5 \pm 0.2 \mathrm{Oe})$, with a rise time of $150 \mathrm{ps}$. A constrained Bloch-Bloembergen model (Ref. 18) was used to fit the data with parameters $T_{2}^{(0)}=1.5 \mu \mathrm{s}, \quad \alpha_{0}=0.0082$. The graphs also show the absolute value of $\mathbf{M}$ (solid circles) for the individual measurements. 


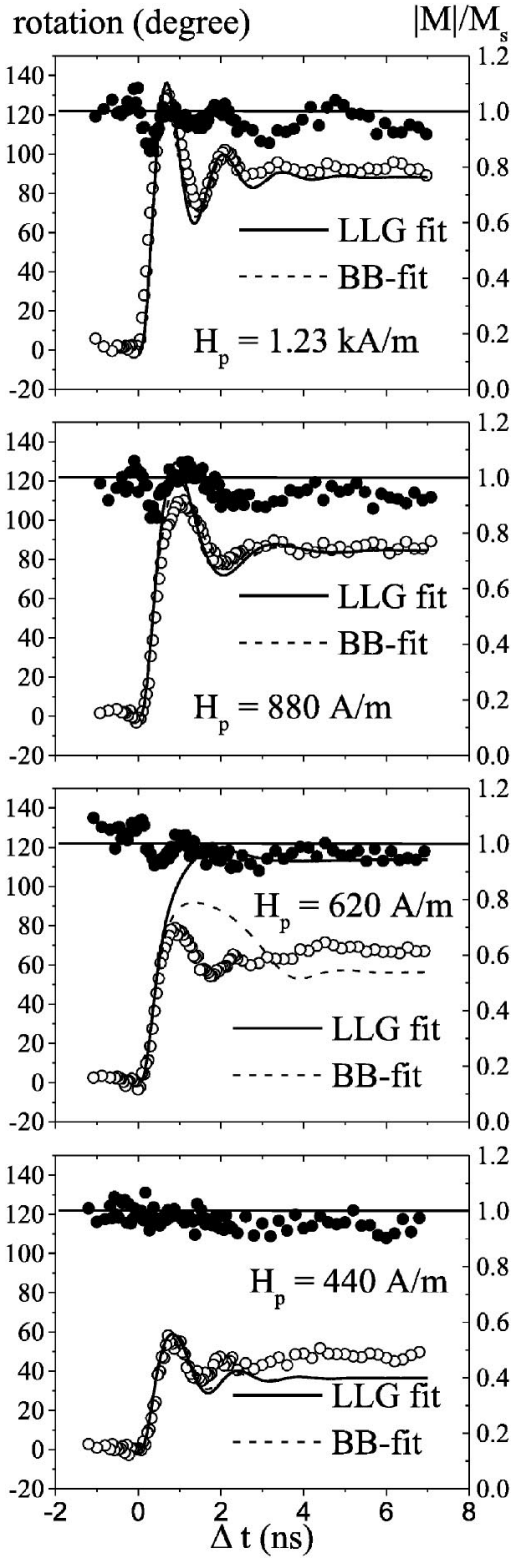

FIG. 4. In-plane vector-resolved measurements for different pulse field strengths (open circles). The bias field was $H_{b}=20 \pm 10 \mathrm{~A} / \mathrm{m}(0.2 \pm 0.1 \mathrm{Oe})$. The field pulses are $H_{p}=1230 \pm 20,880 \pm 20,620 \pm 10$, and $440 \pm 10 \mathrm{~A} / \mathrm{m}$ $(15.5 \pm 0.2,11.7 \pm 0.2,7.9 \pm 0.1$, and $5.5 \pm 0.1 \mathrm{Oe})$. The data were fit using the CBB model with parameters: $T_{2}^{(0)}=1.5 \mu \mathrm{s}, \alpha_{0}=0.0082$ and using the LLG model with $\alpha=0.012$. $|\mathbf{M}|$ is given by the solid circles.

Figs. 3 and 4. The values obtained for $|\mathbf{M}|$ show some decrease during the very first large deflection from the initial direction. The magnitude of this reduction gets smaller with increasing bias field and disappears in the noise for $H_{b}$ $=810 \mathrm{~A} / \mathrm{m}$. This reduction of $|\mathbf{M}|$ for large-angle deflections originates partly from 52 ps jitter (due to the pulse generator circuit) of the pulse-field arrival with respect to the probelaser pulse. We can show, by assuming a linear response (i.e., $\Delta \phi=d \phi / d t \cdot \Delta t)$ that the jitter results in a decrease of $|\mathbf{M}|$ on the order of a few percent, increasing with increasing $d \phi / d t$ and hence increasing with $d \mathbf{M} / d t$. However, after subtraction of the jitter contribution, a reduction of $|\mathbf{M}|$ remains present for small bias fields and large $d \phi / d t$, as shown in Fig. 5. Figure 5 shows a plot of the absolute value of $\mathbf{M}$ at its

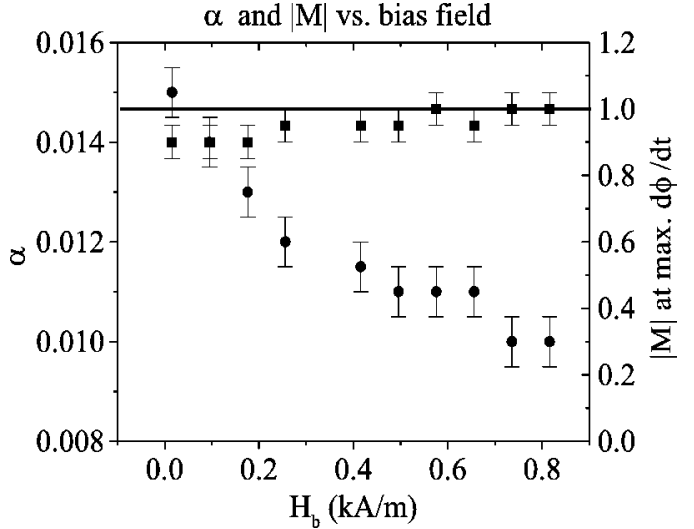

FIG. 5. Damping parameter $\alpha$ and $|\mathbf{M}|$ as a function of applied bias field. The filled squares show the absolute value of $\mathbf{M}$ at the point in the time trace where the rate of magnetization change $d \phi / d t$ is maximum. The damping parameter $\alpha$ (filled circles) was obtained by fits of the data using the LLG model.

maximum dip (after subtraction of the jitter) and the extracted Gilbert damping parameter $\alpha$ versus $H_{b}$. Fitting of the data to the LLG model is used to determine $\alpha$. $|\mathbf{M}|$ decreases by about $10 \%$, on the order of the noise level. However, $\alpha$ increases from 0.010 at $H_{b}=800 \mathrm{~A} / \mathrm{m}$ to a value of 0.015 at $H_{b}=20 \mathrm{~A} / \mathrm{m}$, being an increase of $50 \%$. Therefore, a significant correlation between $\alpha$ and the generation of spin waves cannot be inferred.

In addition to fitting with LLG, we also used the constrained version of the Bloch-Bloembergen $(\mathrm{CBB})$ to fit the data. ${ }^{18}$ Fitting to the $\mathrm{CBB}$ model also allows for extraction of important system parameters, such as the anisotropy and damping constant. This model accounts for increases in the Gilbert damping parameter $\alpha$ for small bias fields as the result of a component of the transverse relaxation rate of the spin system that is invariant with changing ground-state energy. For small-angle motion and small damping, the CBB equation is approximately equivalent to the LLG equation after using the substitution: ${ }^{18}$

$$
\alpha=\alpha_{0}+\left\lfloor|\gamma| \mu_{0} H_{m} T_{2}^{(0)}\right\rfloor^{-1},
$$

where $\alpha_{0}$ is the spectroscopic damping parameter, $1 / T_{2}^{(0)}$ is the critical transverse relaxation rate, and $H_{m}$ is the effective field component along $\mathbf{M}$. In the case of the CBB model, the transverse damping torque is proportional to the transverse component of angular momentum. The total damping torque is determined by application of the additional constraint that $d M / d t=0$. In the limit of large applied fields, Eq. (2) is approximated by $\alpha \approx \alpha_{0}$.

Fits to the data in Figs. 3 and 4 were performed using the CBB equation ${ }^{18}$

$$
\frac{d \vec{M}}{d t}=\gamma \mu_{0} \vec{M} \times \vec{H}+\frac{1}{T_{2} \vec{M} \cdot \vec{H}} \vec{M} \times(\vec{M} \times \vec{H}),
$$




$$
\frac{1}{T_{2}}=\frac{1}{T_{2}^{(0)}}+\frac{\alpha_{0} \gamma \mu_{0} U_{0}}{M_{S}}
$$

and $U_{0}$ is the ground-state energy density of the magnetization. The fitted anisotropy energy was $260 \mathrm{~J} / \mathrm{m}^{3}$, which corresponds to $H_{k}=520 \mathrm{~A} / \mathrm{m}$. In these calculations a correction for the finite CPW width has been included. The value obtained is more than twice the value determined from static measurements. This result confirms results obtained by Lopusnik et al. ${ }^{19}$ where a rotatable contribution to the anisotropy was observed for sputtered permalloy films.

The fits to all the data were obtained by use of constant damping parameters $T_{2}^{(0)}=1.5 \mu$ s and $\alpha_{0}=0.0082$. The values for the damping were chosen as best values for fitting of all the data with the least amount of error. From these fits to the CBB model, a clear increase in the relaxation rate can be inferred for decreasing bias fields. The fits generally agree well with the LLG fits mentioned earlier. Figure 4 shows a comparison of fits to the data using the LLG and CBB models, where the LLG damping was locked to $\alpha=0.012$. A discrepancy is found when the pulse field approximately equals the anisotropy of the system $\left(H_{p}=620 \pm 10 \mathrm{~A} / \mathrm{m}, H_{k}\right.$ $=520 \pm 40 \mathrm{~A} / \mathrm{m})$; neither of the two models fits the data well. The LLG model underestimates the damping. The CBB model at least predicts that switching does not occur, as is observed. The failure to switch for the CBB simulation is due to the enhancement of the effective damping when $\vec{M} \cdot \vec{H} \approx 0$. Such viscous response is to be expected whenever the apex in the precessional oscillations is close to the hard axis direction. (It is improbable that $\vec{M} \cdot \vec{H}=0$ is strictly obtained in a thin film geometry because the demagnetizing fields induced by the shape anisotropy require that $\vec{M} \cdot \vec{H}$ $\neq 0$ unless the magnetization is at the apex of the in-plane precessional motion.)

To verify the increase in LLG damping $\alpha$ with decreasing $H_{b}$ observed using MSHG, additional PIMM measurements were carried out for the same magnetic film by use of a PIMM with a step pulse field of $H_{p}=320 \mathrm{~A} / \mathrm{m}(4 \mathrm{Oe})$, by means of a CPW with a $500 \mu \mathrm{m}$ center conductor width. The PIMM measurements, presented in Fig. 6, also show a clear increase in LLG damping $\alpha$ with decreasing bias fields. In addition, the extracted values for $\alpha$ obtained with the PIMM are somewhat lower than those extracted from the vectorresolved MSHG data. The difference between the two measurements might result from the much larger excitation angles for the vector resolved measurements. Fitting the PIMM data with Eq. (2) yields $T_{2}^{(0)}=2.1 \pm 0.1 \mu$ s and $\alpha_{0}$ $=0.0083 \pm 0.0005$. The field originating from the anisotropy was dynamically determined to be $430 \mathrm{~A} / \mathrm{m}$. The similarity of the damping obtained for local MSHG measurements and macroscopic PIMM experiments suggests that the increase in the LLG $\alpha$ for small $H_{b}$ is not the result of inhomogeneous effects. If the damping change were simply due to dispersion of the internal fields over a macroscopic length scale, we would not expect to observe the same variation in damping with bias field when making a local measurement of the magnetization dynamics. In addition, we have also measured the damping as a function of temperature. From these mea-

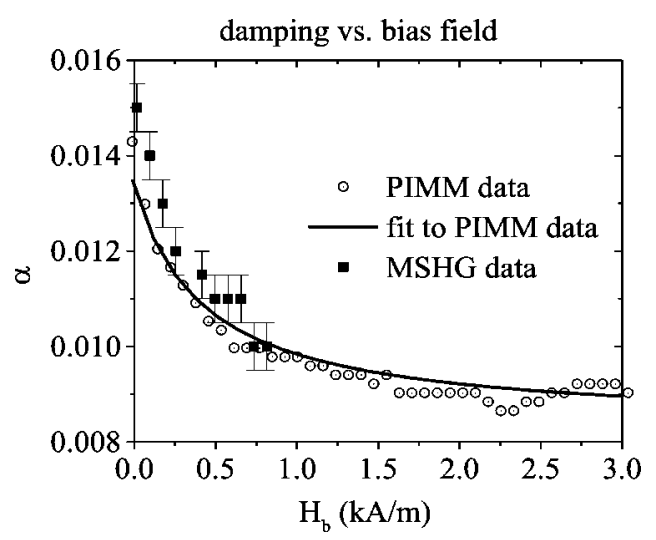

FIG. 6. Extraction of the damping parameter from PIMM data for a bias field dependent measurement and a steplike excitation pulse $H_{p}$ $=320 \mathrm{~A} / \mathrm{m}(4 \mathrm{Oe})$ (open circles). The squares show the extracted Gilbert damping parameter of the vector resolved data. The solid line shows the CBB fit to the PIMM data with a dynamically determined anisotropy of $430 \mathrm{~A} / \mathrm{m}$.

surements, thermally induced spin fluctuations as an origin for the inverse field proportionality of the damping can be excluded, as no significant temperature dependence of the increase in damping at low bias fields could be found.

\section{CONCLUSION}

Pump-probe MSHG can be used to determine the magnetization dynamics of a patterned magnetic element excited by a magnetic field pulse. Using this technique, we can clearly determine whether changes in the damping parameter and the homogeneity of the precession response are correlated, as inferred from the absolute value of $\mathbf{M}$ and the timetrace of the response. $|\mathbf{M}|$ did not decrease for small bias fields when the field pulses were strong enough to saturate the system along its hard axis. This is in contrast to the measurements by Silva et al. ${ }^{5}$ when a sample was biased along its hard axis, and also in contrast to the $180^{\circ}$ switching experiments of Hiebert and co-workers ${ }^{20}$ in which $\mathbf{M}$ was forced to break into domains while switching. In the present case, the magnetic system stays coherent, and magnonmagnon scattering processes do not dominate the damping. Even at very large excitation angles and low bias fields, where a significant increase in $\alpha$ is required to fit the data, the uniform mode is preserved. We conclude that magnonmagnon relaxation is not sufficient to explain pulsed reorientation of thin metal films.

\section{ACKNOWLEDGMENTS}

The authors would like to thank H.A.M. van den Berg for his fruitful contribution to this work, Tony Kos for assistance in preparing the devices for the pulse-generator measurements, and Radek Lopusnik for many helpful discussions. This work was partially supported by the Stichting Fundamenteel Onderzoek der Materie (FOM) and by the European "DYNAMICS" project. This work was supported by a fellowship within the Postdoc-Programme of the German Academic Exchange Service (DAAD). Work partially supported by NIST, an agency of the U.S. government. 
${ }^{1}$ T. M. Crawford, T. J. Silva, C. W. Teplin, and C. T. Rogers, Appl. Phys. Lett. 74, 3386 (1999).

${ }^{2}$ W. K. Hiebert, A. Stankiewcz, and M. R. Freeman, Phys. Rev. Lett. 79, 1134 (1997).

${ }^{3}$ C. Patton, High Frequency Relaxation Phenomena in ferromagnetic systems-Phenomenology and Physics, Invited talk Amsterdam Intermag Conference, 1 May 2002

${ }^{4}$ Th. Gerrits, H. A. M. van den Berg, J. Hohlfeld, L. Bär, and Th. Rasing, Nature (London) 418, 509 (2002).

${ }^{5}$ T. J. Silva, P. Kabos, and M. R. Pufall, Appl. Phys. Lett. 81, 1 (2002).

${ }^{6}$ G. E. Ballentine, W. K. Hiebert, A. Stankiewicz, and M. R. Freeman, J. Appl. Phys. 87, 6830 (2000).

${ }^{7}$ M. Bauer, R. Lopusnik, J. Fassbender, and B. Hillebrands, Appl. Phys. Lett. 76, 2758 (2000).

${ }^{8}$ T. L. Gilbert, Phys. Rev. 100, 1243 (1955).

${ }^{9}$ M. Sparks, Ferromagnetic-Relaxation Theory (McGraw-Hill, New York, 1964), p. 166.

${ }^{10}$ G. T. Rado and H. Suhl, Magnetism I (Academic, New York, 1963), p. 552.
${ }^{11}$ J. P. Nibarger, R. Lopusnik, and T. J. Silva, Appl. Phys. Lett. 82, 2112 (2003).

${ }^{12}$ Th. Rasing, J. Magn. Soc. Jpn. 20, 13 (1996).

${ }^{13}$ T. M. Crawford, C. T. Rogers, T. J. Silva, and Y. K. Kim, IEEE Trans. Magn. 32, 4087 (1996).

${ }^{14}$ In the case of thin magnetic films the magnetization stays mostly in the film plane during precessional motion. Therefore, only the in-plane magnetization components are used for the determination of $|\mathbf{M}|$.

${ }^{15}$ T. J. Silva, C. S. Lee, T. M. Crawford, and C. T. Rogers, J. Appl. Phys. 85, 7849 (1999).

${ }^{16}$ T. J. Klemmer, K. A. Ellis, and B. van Dover, J. Appl. Phys. 87, 5846 (2000).

${ }^{17}$ P. Kabos, A. B. Kos, and T. J. Silva, J. Appl. Phys. 87, 5980 (2000).

${ }^{18}$ T. J. Silva and Th. Gerrits (unpublished).

${ }^{19}$ R. Lopusnik, J. P. Nibarger, T. J. Silva, and Z. Celinski, Appl. Phys. Lett. 83, 96 (2003).

${ }^{20}$ B. C. Choi, M. Belov, W. K. Hiebert, G. E. Ballentine, and M. R. Freeman, Phys. Rev. Lett. 86, 728 (2001). 